\title{
INDEPENDANCE ALGEBRIQUE DES DERIVEES D'UNE PERIODE DU MODULE DE CARLITZ
}

\section{LAURENT DENIS}

(Received 16 June 1999; revised 28 September 1999)

Communicated by W. W. Chen

\begin{abstract}
We prove here that the $p-1$ first derivatives of the fundamental period of the Carlitz module are algebraically independent. For that purpose we will show how to use Mahler's method in this situation. 2000 Mathematics subject classification: primary 11G09, $11 \mathrm{~J} 85$.
\end{abstract}

\section{Position du problème}

On désigne par $A=F_{q}[T]$ l'anneau des polynômes en une variable à coefficients dans le corps fini $F_{q}$ de caractéristique $p$, par $k=F_{q}(T)$ son corps des fractions, par $k_{\infty}=F_{q}(1 / T)$ le complété de $k$ pour la valuation $(1 / T)$-adique $v$ normalisée par $v(T)=-1$, que l'on prolonge à une clôture algébrique $\bar{k}$ (resp. $\bar{k}_{\infty}$ ) de $k$ (resp. $k_{\infty}$ ) et par $C$ le complété de $\bar{k}_{\infty}$. On notera $|\alpha|=q^{-v(\alpha)}=q^{\operatorname{deg}(\alpha)}$, la valeur absolue d'un élément de $C$ avec la convention $\operatorname{deg}(0)=-\infty$.

Définitions-propriétés Le module de Carlitz est la donnée du groupe additif $G_{a}$ et de l'homomorphisme injectif et $F_{q}$-linéaire d'anneaux $\Phi: A \rightarrow \operatorname{End}\left(G_{a}\right)$ défini par:

$$
\Phi(T)=T F^{0}+F
$$

où $F$ est le Frobenius relatif à $q$.

Il existe alors une unique fonction exponentielle $e(z)$, caractérisée par les propriétés:

$$
\begin{aligned}
e(0) & =0, \quad d / d z(e(z))=1 ; \\
\text { pour tout } z \in C: \quad e(T z) & =\Phi(T)(e(z))=T e(z)+e(z)^{q} .
\end{aligned}
$$

(C) 2000 Australian Mathematical Society $0263-6115 / 2000 \$ A 2.00+0.00$ 
Rappelons qu'il existe alors un élément $\pi_{q} \in k_{\infty}$ tel que $\operatorname{Ker} e(z)=A(T-$ $\left.T^{q}\right)^{1 /(q-1)} \pi_{q}([\mathrm{C}])$. Nous avons vu ([D3]) comment la méthode de Mahler pouvait conduire à des propriétés d'indépendance algébrique pour les différents $\pi_{q i}(i$ entier $\geq 1$ ) existants dans $k_{\infty}$. Ici nous travaillerons avec un seul de ces nombres et poserons simplement $\pi_{q}=\pi$. Les $p$ premières dérivées de $\pi$ notées $\pi^{(i)}(0 \leq i \leq p-1)$ sont des éléments de $k_{\infty}$ qui apparaîssent comme des combinaisons linéaires de périodes de certains $T$-modules (voir [D1]). Il a été prouvé dans ([D1]) que $1, \pi, \ldots, \pi^{(p-1)}$ sont linéairement indépendants sur $\bar{k}$, ce résultat a été étendu par $D$. Brownawell aux $q$ premières dérivées divisées de $\pi$ ([B]) puis par Brownawell et Denis ([BD]) à un nombre arbitraire de dérivées divisées. Dans ([D2]) nous avons par ailleurs prouvé que $\pi$ et $\pi^{\prime}$ sont algébriquement indépendants sur $k$. Nous prouvons ici le théorème suivant.

THÉORÈME 1. Les $p$ éléments de $k_{\infty}: \pi, \ldots, \pi^{(p-1)}$; sont algébriquement indépendants sur $k$.

Au Paragraphe 2 nous démontrerons tout d'abord un analogue d'un résultat d'indépendance algébrique de Becker concernant la méthode de Mahler sur les extensions finies de $k$ (jusqu'ici il semble que cette méthode n'a été explorée que pour les séries à coefficients dans un corps fini ([B2]). C'est un cas particulier de ce théorème qui était déjà utilisé dans ([D3]). Enfin le Théorème 1 est démontré au Paragraphe 3.

\section{Un peu de méthode de Mahler}

Nous n'aurons besoin que du théorème d'indépendance algébrique utilisé dans ([D3]). Toutefois, nous choisirons de donner la preuve d'un analogue plus complet du théorème principal de ([B1]). La preuve ne s'écarte de ([B1]) que pour des questions d'ultramétricité et est donnée pour la tranquillité du lecteur.

DÉFINITION 1. Soit $P(z)=\sum_{i=0}^{d} a_{i} z^{i}$ un polynôme de $C[z]$ de degré $d \geq 2, \rho$ l'unique réel positif tel que $\left|a_{d}\right|=\max _{0 \leq i \leq d-1}\left\{\left|a_{i} \rho^{d-1}\right|,\left|\rho^{d-1}\right|\right\}$. On définit alors l'opérateur $\mathbf{T}$ de $V_{\mathbf{T}}=\{z \in C \backslash 0<|z|<\rho\}$ dans lui même par $\mathbf{T}(z)=[P(1 / z)]^{-1}$ et par $\mathbf{T}^{s}$ son $s$-ième itéré.

Nous allons démontrer le théorème suivant:

THÉORÈME 2. Soit $f_{1}, \ldots, f_{m}$ des fonctions de $C$ dans $C$ ayant un développement de Taylor convergeant dans un voisinage $V$ de zéro de type $|z|<r \leq 1$ et à coefficients dans une extension finie $k_{1}$ de $k$. Supposons que ces fonctions sont algébriquement indépendantes sur $C(z)$ et qu'il existe des fractions rationnelles $a_{i}(z)$ et $b_{i}(z)(1 \leq$ 
$i \leq m)$ dans $k_{1}(z)$ telles que

$$
f_{i}(z)=a_{i}(z) f_{i}(\mathbf{T}(z))+b_{i}(z), \quad 1 \leq i \leq m .
$$

Alors pour tout $\alpha$ algébrique non nul appartenant à $V \cap V_{\mathrm{T}}$ tel que pour tout entier $n$, $\mathbf{T}^{n}(\alpha)$ n'est zéro d'aucun $a_{i}(z)$ et pôle d'aucun $b_{i}(z)$, les nombres $f_{1}(\alpha), \ldots, f_{m}(\alpha)$ sont algébriquement indépendants sur $k$.

Etablissons les quelques lemmes préliminaires nécessaires et analogues à ceux de ([B1]).

LEMME 1. Pour tout $z$ appartenant à $V_{\mathbf{T}}$ et tout entier naturel $n$ :

$$
\operatorname{deg}\left(\mathbf{T}^{n}(z)\right)=d^{n}\left(\operatorname{deg}(z)+\frac{\operatorname{deg}\left(a_{d}\right)}{1-d}\right)-\frac{\operatorname{deg}\left(a_{d}\right)}{1-d}
$$

PREUVE. $\operatorname{deg}(P(1 / z))=\operatorname{deg}\left(\sum_{i=0}^{d} a_{i} z^{d-i}\right)-d \operatorname{deg}(z)=\operatorname{deg}\left(a_{d}\right)-d \operatorname{deg}(z)$, d'où $\operatorname{deg}(\mathrm{T}(z))=d \operatorname{deg}(z)-\operatorname{deg}\left(a_{d}\right)$. On conclut en calculant le $s$-ième terme de la suite récurrente $u_{n+1}=d u_{n}-\operatorname{deg}\left(a_{d}\right)$ qui est le translaté d'une suite géométrique de raison $d$.

Pour $P \in \bar{k}\left[X_{1}, \ldots, X_{m}\right]$, on définit sa hauteur $h(P)$ comme étant la hauteur logarithmique absolue de Weil définie par l'ensemble de ses coefficients. Pour tout corps $L$ contenant les coefficients $x_{\mathrm{i}}$ de $P$ :

$$
h(P)=\sum_{v} \frac{d(v) \max \left\{0,-v\left(x_{i}\right)\right\}}{[L: k]}
$$

où la somme est étendue à toutes les places de $L, d(v)$ désigne le degré résiduel sur $F_{q}$ en la place $v$, normalisée par le fait que $v(L)$ soit l'ensemble constitué des entiers relatifs et de $\{+\infty\}$ et le maximum est pris sur tous les coefficients de $P$. Soit $K$ une extension finie de $k$. On déduit aisément du Théorème 2 de ([P]) le critère d'indépendance algébrique suivant.

LEMME 2 (Philippon). Soit $\theta=\left(\theta_{1}, \ldots, \theta_{m}\right)$ un élément de $C^{m}$, supposons qu'on ait quatre fonctions croissantes $\delta, \sigma, \varepsilon$ et $\rho$ de $N$ à valeurs dans les réels plus grands que 1 et pour tout entier $n$, une famille de polynômes $Q_{1, n}, \ldots, Q_{u(n), n} \in$ $K\left[X_{1}, \ldots, X_{m}\right]$ de degré total $\leq \delta(n)$, de hauteur $\leq \sigma(n)$ sans zéro commun dans la boule $\operatorname{deg}(z-\theta) \leq-\rho(n)$ et satisfaisnat

$$
-\infty<\max _{1 \leq j \leq u(n)} \operatorname{deg}\left(Q_{j, n}(\theta)\right) \leq-\varepsilon(n) .
$$


On suppose en outre $\delta(n) \leq \sigma(n), \varepsilon(n) \leq \rho(n+1)$, que pour un entier $t$, on ait $(\delta+\sigma)(n)$ qui tend vers $+\infty$ et $\left[\varepsilon /(\delta+\sigma) \delta^{t}\right](n)$ qui croissent avec $n$, et que pour tout $c$ réel suffisamment grand, ne dépendant que de $K$ et $\theta$, on ait:

$$
\varepsilon(n)^{t+1}>c(\delta+\sigma)(n) \delta^{t-1}(n)\left[\varepsilon(n+1)^{t}+\rho(n+1)^{t}\right] .
$$

Alors le degré de transcendance sur $K$ de $K\left(\theta_{1}, \ldots, \theta_{m}\right)$ est supérieur à $t$.

Dans les lemmes qui suivent, on se place sous les hypothèses du Théorème 2. On désigne par $C_{1}, C_{2}, \ldots$ des réels positifs ne dépendant que de $\alpha$, des fonctions $f_{i}, a_{i}$, $b_{i}(1 \leq i \leq m)$ et du corps $k_{1}$ et par $D_{1}(N), D_{2}(N), \ldots$ des fonctions ne dépendant que des paramètres précédents et de $N$.

LEMME 3. Soit $\mathbf{f}(z)=\left(f_{1}(z), \ldots, f_{m}(z)\right)$ un $m$-uplet de fonctions satisfaisant les hypothèses du Théorème 2 et $N$ un entier naturel $\geq C_{1}$, il existe un polynôme $R(z, \mathbf{X}) \in A\left[z, X_{1}, \ldots, X_{m}\right]$ tel que:

(a) les $m+1$ degrés partiels de $R$ sont $\leq N$;

(b) $E_{N}(z)=R(z, \mathbf{f}(z))$ n'est pas indentiquement nulle et s'annule en zéro à un ordre $\nu(N) \geq C_{2} N^{m+1}$.

PREUVE. Il s'agit de résoudre un système linéaire ayant plus d'inconnues que d'équations, $E_{N}(z)$ est non nulle car.Jes fonctions $f_{1}(z), \ldots, f_{m}(z)$ sont algébriquement indépendantes.

Soit $B$ l'anneau des entiers sur $A$ de $k_{1}, c(z) \in B[z]$ tel que $c(z) a_{i}(z), c(z) b_{i}(z)$ soient dans $B[z]$ pour $1 \leq i \leq m$. Posons $\mathbf{p}(z)=D z^{d} P(1 / z)$ où $D$ est un dénominateur appartenant à $A$ des coefficients du polynôme $P$. On considère alors $C_{3}$ un majorant entier naturel des degrés des polynômes $c(z), c(z) a_{i}(z), c(z) b_{i}(z)$ (pour $1 \leq i \leq m), p(z), \mathbf{p}(z)$ et $C_{4}$ un majorant de leur hauteur et de $D$.

On définit donc (toujours comme dans ([B2])), une suite de fractions rationnelles $R_{n}(z, \mathbf{X}) \in A(z)\left[X_{1}, \ldots, X_{m}\right]$ par: $R_{0}(z, \mathbf{X})=R(z, \mathbf{X})$ et la relation de récurrence

$$
R_{n}(z, \mathbf{X})=c(z)^{N} \mathbf{p}(z)^{N d_{n-1}} R_{n-1}(\mathbf{T}(z), \mathbf{a}(z) \mathbf{X}+\mathbf{b}(z))
$$

où les lettres grasses $\mathbf{a}$ et $\mathbf{b}$ désignent les vecteurs coordonnées dont les composantes sont les lettres non grasses correspondantes et $d_{n}=C_{3}\left(1+d+\cdots+d^{n-1}\right)+d^{n}$. On prouve alors les estimations suivantes.

LEMME 4. (a) $\operatorname{deg}_{\mathrm{X}} R_{n}(z, \mathrm{X}) \leq N$;

(b) $\operatorname{deg}_{z} R_{n}(z, X) \leq d_{n} N \leq 3 C_{3} d^{n} N$ et $R_{n}(z, X) \in A(z)\left[X_{1}, \ldots, X_{m}\right]$;

(c) $h\left(R_{n}(z, \mathbf{X})\right) \leq h\left(R_{0}(z, \mathbf{X})\right)+C_{6} d^{n} N$; 
(d) soit $\left(\alpha_{\mathrm{j}, n}\right)_{\mathbf{j} \in N^{m+1}}$ les coefficients du polynôme $R_{n}(z, \mathbf{X})$ pour $n \geq D_{1}(N)$, $\max _{\mathbf{j}, n}$ $\operatorname{deg}\left(\alpha_{\mathrm{j}, n}\right) \leq C_{7} d^{n} N$.

PREUVE. (a) et (b) arrivent aisément par récurrence. (c) et (d) la formule de récurrence montre qu'il existe un réel $C_{5}$ tel que pour toute valeur absolue $\mid \|_{v}$ de $K$,

$$
\max \left|\alpha_{\mathbf{j}, n}\right|_{v} \leq C_{5}^{\left(d^{n-1} N+N\right)} \max \left|\alpha_{\mathbf{j}, n-1}\right|_{v}
$$

il s'ensuit par sommation sur les places de $K$ :

$$
h\left(R_{n}(z, \mathbf{X})\right) \leq h\left(R_{n-1}(z, \mathbf{X})\right)+C_{5}\left(d^{n} N+N\right)
$$

d'où le résultat.

LEMME 5. En conservant les notations des Lemmes 3 et 4 ainsi que celles du Théorème 2, pour tout $N \geq C_{7}$ et $n \geq D_{2}(N)$ on dispose des inégalités:

$$
-C_{8} d^{n} \nu(N) \leq \operatorname{deg}\left(R_{n}(\alpha, \mathbf{f}(\alpha))\right) \leq-C_{9} d^{n} v(N) .
$$

PREUVE. Comme dans ([B1]), la preuve vient du Lemme 1 précédent et du fait que $R_{n}(\alpha, \mathbf{f}(\alpha))=E_{N}\left(\mathbf{T}^{n}(\alpha)\right) \prod_{j=0}^{n-1}\left[c\left(\mathbf{T}^{j}(\alpha)\right) \mathbf{p}\left(\mathbf{T}^{j}(\alpha)\right)^{d_{n-j-1}}\right]^{N}$.

Procédons alors à la preuve du Théorème 2 . On construit la suite de polynômes $Q_{n}(\mathbf{X}) \in B\left[X_{1}, \ldots, X_{m}\right]$ en posant:

$$
Q_{n}(\mathbf{X})=\left(D^{\prime}\right)^{3 C_{3} d^{n} N} R_{n}(\alpha, \mathbf{X}),
$$

où $D^{\prime}$ est un dénominateur de $\alpha$.

Grâce au Lemme 1, pour $n \geq D_{3}(N)$ on $a$ :

$$
\operatorname{deg}_{\mathbf{X}} Q_{n}(\mathbf{X}) \leq N, \quad h\left(Q_{n}(\mathbf{X})\right) \leq C_{10} d^{n} N .
$$

Comme $v(N) \geq N^{m+1}$, le Lemme 4 (d) et les inégalités précédentes entraînent que $Q_{n}(\mathbf{z})$ ne s'annule pas dans la boule

$$
\operatorname{deg}(\mathbf{z}-\mathbf{f}(\alpha)) \leq C_{11} d^{n} v(N)
$$

où on choisit de plus $C_{11}>C_{9}$.

Appliquons alors le Lemme 2 avec $\theta=\left(f_{1}(\alpha), \ldots, f_{m}(\alpha)\right), t=m, u(n)=1$, $\varepsilon(n)=C_{9} d^{n} v(N), \rho(n)=C_{11} d^{n} v(N), \delta(n)=N, \sigma(n)=C_{10} d^{n} v(N)$. Ce choix permet de vérifier toutes les estimations nécessaires pour prouver que $f_{1}(\alpha), \ldots, f_{m}(\alpha)$ sont algébriquement indépendants sur $k$. 


\section{Indépendance de quelques fonctions}

Comme c'est usuellement le cas pour appliquer la méthode de Mahler, nous allons prouver l'indépendance algébrique de quelques fonctions. On utilisera les lemmes suivants.

LEMME 6. Soit $A(X)$ un polynôme de $C[X]$ de degré $<q$ et $f$ une fraction rationnelle dans $C(X)$ telle que:

$$
f\left(X^{q}\right)=f(X)+A(X)
$$

alors $f$ est constante et $A$ est nul.

PREUVE. Si $a$ est un pôle de $f$ alors $a^{1 / q}$ est un pôle de $f\left(X^{q}\right)$ et donc, au vu de l'équation fonctionnelle, également un pôle de $f$. De même pour tout entier $h, a^{1 / q^{n}}$ est encore un pôle de $f$. Comme $f$ n'a qu'un nombre fini de pôles, il existe un entier $j \geq 1$ tel que $a=a^{q^{j}}$ et donc $a \in F_{q^{j}}$. L'itération de la relation fonctionnelle prouve que $f\left(X^{q^{j}}\right)=f(X)+A_{j}(X)$ où $A_{j}(X)$ est un polynôme. La décomposition en éléments simples sur $C$ de $f$ montre que le terme polaire d'ordre maximal dans $f(X)$ est de la forme $c /(X-a)^{t}$, et que dans $f\left(X^{q^{j}}\right)$ il est $c /(X-a)^{t q^{j}}$. Donc $f$ n'a aucun pôle à distance finie, c'est un polynôme, la conclusion du lemme résulte de l'examen du degré des deux membres de l'équation.

LEMME 7. Soit $A(X)$ un polynôme de $C[X], b$ un élément de $C$ n'appartenant pas à $\bar{F}_{q}$ tel que $A(b) \neq 0, t$ un entier naturel non nul. Il n'existe aucune fraction rationnelle $f$ telle que:

$$
f\left(X^{q}\right)=f(X)+\frac{A(X)}{(X-b)^{t}} .
$$

Preuve. Si $a$ est un pôle de $f$ alors $a^{1 / q}$ est un pôle de $f\left(X^{q}\right)$ et donc, au vu de l'équation fonctionnelle, un pôle de $f$ si $b \neq a^{1 / q}$. De proche en proche, si $a$ est tel que pour tout entier $h$ supérier à $1, b \neq a^{1 / q^{h}}$, on démontre comme au Lemme 6 que $a$ n'est pas un pôle de $f$. Si maintenant $a$ est un pôle de $f$ différent de $b$, d'après l'équation, $a$ est un pôle de $f\left(X^{q}\right)$ donc $a^{q}$ est un pôle de $f$. De proche en proche, si $a$ est tel que pour.tout entier $h, b \neq a^{q^{h}}$, on démontre comme au Lemme 6 que $a$ n'est pas un pôle de $f$. Donc si $a$ est un pôle de $f$, il existe un entier $j \geq 1$ tel que $b=a^{1 / q^{j}}$ et il existe un entier $j^{\prime}$ tel que $b=a^{q^{j^{\prime}}}$, c'est absurde car $b$ n'appartient pas à $\bar{F}_{q}$.

Pour tout entier $i \geq 1$ on considère la fonction $f_{i}$ de $C$ dans $C$ analytique dans le disque non circonférencié dont un centre est 0 et de rayon $1 / q$ méromorphe dans le 
disque de rayon 1 et définie par l'expression:

$$
f_{i}(z)=\sum_{h=0}^{+\infty} \frac{z^{i q^{h}}}{\left(1-T z^{q^{h}}\right)^{i}} .
$$

Des fonctions définies par des expressions proches de celles donnant les $f_{i}$ se rencontrent fréquemment en caractéristique zéro (voir par exemple $[\mathrm{B} 2, \mathrm{~N}]$ ). Comme il est clair que $f_{p}(z)=\left[f_{\mathrm{l}}(z)\right]^{p}$, le théorème suivant est le meilleur possible.

THÉORÈME 3. Les $p-1$ fonctions $f_{i}(z)(1 \leq i \leq p-1)$ sont algébriquement indépendantes sur $C(z)$.

PREUVE. Prouvons par récurrence sur l'entier $i \leq p-1$ que les $f_{j}(z)(1 \leq j \leq i)$ sont algébriquement indépendantes sur $C(z)$. Pour $i=1$, indiquons plus généralement qu'une fonction qui a une infinité de pôles est transcendante sur $C(z)$. En effet, si une telle fonction $f(z)$ est algébrique alors il existe un polynôme $P(z)$ dans $C[z]$ tel que $P(z) f(z)$ soit entière algébrique sur $C[z]$. Comme $f(z)$ possède une infinité de pôles, on aboutit à une contradiction en examinant une relation de dépendance intégrale.

Par hypothèse de récurrence, si les fonctions $f_{j}(z)(1 \leq j \leq i)$ sont algébriquement indépendantes sur $C(z)$, il existe un polynôme irréductible $P \in C\left[X_{0}, X_{1}, \ldots, X_{i}\right]-$ $C\left[X_{0}\right]$, engendrant l'idéal des relations de dépendances des fonctions $f_{j}(z)(1 \leq j \leq i)$ sur $C$ tel que pour tout $z$ dans le disque de convergence on ait

$$
P\left(z, f_{1}(z), \ldots, f_{i}(z)\right)=0,
$$

et donc que:

$$
P\left(z^{q}, f_{1}(z)-\frac{z}{(1-T z)}, \ldots, f_{i}(z)-\frac{z^{i}}{(1-T z)^{i}}\right)=0 .
$$

Il existe alors un entier $N$ tel que

$$
\begin{aligned}
(1- & \left.T X_{0}\right)^{N} P\left(X_{0}^{q}, X_{1}-\frac{X_{0}}{\left(1-T X_{0}\right)}, \ldots, X_{i}-\frac{X_{0}^{i}}{\left(1-T X_{0}\right)^{i}}\right) \\
& :=P^{(q)}\left(X_{0}, X_{1}, \ldots, X_{i}\right)
\end{aligned}
$$

soit un polynôme et donc on a un élément $R \in C\left[X_{0}, X_{1}, \ldots, X_{i}\right]$ tel que

$$
P^{(q)}\left(X_{0}, X_{1}, \ldots, X_{i}\right)=R\left(X_{0}, X_{1}, \ldots, X_{i}\right) P\left(X_{0}, X_{1}, \ldots, X_{i}\right) .
$$

La comparaison des degrés en $X_{j}(1 \leq j \leq i)$ dans cette relation prouve qu'en fait $R \in C\left[X_{0}\right]$. Nous allons prouver par l'absurde qu'il n'existe aucun polynôme satisfaisant ce type d'équation qui ne soit pas dans $C\left[X_{0}\right]$.

Soit l'anneau $E=\bigcup_{h \geq 0} C\left[X_{0}^{1 / p^{h}}\right], U=\bigcup_{h \geq 0} C\left(X_{0}^{1 / p^{h}}\right)$ son corps des fractions. Considérons $Q$ un polynôme de $E\left[X_{1}, \ldots, X_{i}\right]$, de degré total en $\left(X_{1}, \ldots, X_{i}\right)$ strictement positif et minimal, satisfaisant à la propriété $(\mathbf{P})$ : 
(P) il existe un élément $R\left(X_{0}\right) \in U$ tel que:

$Q\left(X_{0}^{q}, X_{1}-\frac{X_{0}}{\left(1-T X_{0}\right)}, \ldots, X_{i}-\frac{X_{0}^{i}}{\left(1-T X_{0}\right)^{i}}\right)=R\left(X_{0}\right) Q\left(X_{0}, X_{1}, \ldots, X_{i}\right)$.

Pour tout entier $j(1 \leq j \leq i)$ la dérivée de $Q$ par rapport à $X_{j}$ satisfait encore à la propriété $(\mathbf{P})$ et est de degré total strictement inférieur à celui de $Q$. Il s'ensuit que $Q$ est de la forme:

$$
Q\left(X_{0}, X_{1}, \ldots, X_{i}\right)=\sum_{j=1}^{i} c_{j} X_{j}+\sum_{\mathbf{j}} c_{\mathbf{j}} X_{1}^{p j_{1}} \cdots X_{i}^{p j_{i}}
$$

où $\mathbf{j}=\left(j_{1}, \ldots, j_{i}\right)$ et les $c_{j}, c_{\mathbf{j}}$ sont dans $E$.

Comme $U$ est stable par extraction de racines $p$-ièmes, l'hypothèse de minimalité du degré de $Q$, prouve que les $c_{j}$ ne sont pas tous nuls, appelons $c_{j 0}$ un tel coefficient non nul. En examinant les termes de degré partiels 1 , il vient: $c_{j 0}\left(X_{0}^{q}\right)=R\left(X_{0}\right) c_{j 0}\left(X_{0}\right)$. De même, si j est un multi-indice correspondant à un terme du degré total de $Q$, on a: $c_{\mathrm{j}}\left(X_{0}^{q}\right)=R\left(X_{0}\right) c_{\mathrm{j}}\left(X_{0}\right)$. On a alors $c_{\mathrm{j}}\left(X_{0}^{q}\right) / c_{j 0}\left(X_{0}^{q}\right)=c_{\mathrm{j}}\left(X_{0}\right) / c_{j 0}\left(X_{0}\right)$, qui est une égalité de fractions rationnelles de $C\left(X_{0}^{1 / p^{m}}\right.$ ) (pour un certain $m$ ). On en déduit par le Lemme 6 que $c_{\mathrm{j}}\left(X_{0}\right) / c_{j 0}\left(X_{0}\right)$ est dans $C$. Prouvons par récurrence descendante sur la longueur $n$ d'un indice quelconque $\mathbf{j}$ que $c_{\mathrm{j}}\left(X_{0}\right) / c_{j 0}\left(X_{0}\right)$ est dans $C$. C'est déjà vu pour $\mathbf{j}$ maximal. Soit $\mathbf{i}$ de longueur $\leqslant n$, la propriété $(\mathbf{P})$ entraîne une identité du type:

$$
c_{\mathrm{i}}\left(X_{0}^{q}\right)+\sum_{\mathrm{j}>\mathbf{i}} B(\mathbf{j}, \mathbf{e})\left(\frac{X_{0}}{\left(1-T X_{0}\right)}\right)^{e_{1}} \cdots\left(\frac{X_{0}}{\left(1-T X_{0}\right)}\right)^{i e_{i}} c_{\mathrm{j}}\left(X_{0}^{q}\right)=R\left(X_{0}\right) c_{\mathrm{i}}\left(X_{0}\right)
$$

où les $B(\mathbf{j}, \mathbf{e})$ sont des produits de coefficients du binôme et les $e_{s}$ des entiers plus petits que les $j_{s}(1 \leq s \leq i)$.

Divisant les deux membres de la relation par $c_{j 0}\left(X_{0}^{q}\right)=R\left(X_{0}\right) c_{j 0}\left(X_{0}\right)$, et utilisant l'hypothèse de récurrence, on en sort

$$
\frac{c_{\mathrm{i}}\left(X_{0}^{q}\right)}{c_{j 0}\left(X_{0}^{q}\right)}+\sum_{\mathbf{j}>\mathbf{i}} B(\mathbf{j}, \mathbf{e})\left(\frac{X_{0}}{\left(1-T X_{0}\right)}\right)^{e_{1}} \cdots\left(\frac{X_{0}}{\left(1-T X_{0}\right)}\right)^{i e_{i}} d_{j}=\frac{c_{\mathbf{i}}\left(X_{0}\right)}{c_{j 0}\left(X_{0}\right)}
$$

où les $d_{\mathrm{j}}$ sont dans $C$. La fraction rationnelle définie par la sommation ci dessus est de degré négatif en $X_{0}$, c'est donc une constante ou une fraction rationnelle dont le seul pôle est $1 / T$. Le Lemme 7 (appliqué dans l'anneau $C\left[X_{0}^{1 / p^{m}}\right]$ pour un certain $m$ ) conclut au fait que $c_{\mathrm{i}} / c_{j 0}$ est dans $C$.

Il s'ensuit que $Q\left(X_{0}, X_{1}, \ldots, X_{i}\right)=c_{j 0}\left(X_{0}\right)\left(\sum_{j=1}^{i} h_{j} X_{j}+\sum_{\mathrm{j}} h_{\mathrm{j}} X_{1}^{p j_{1}} \ldots X_{i}^{p j_{i}}\right)$ avec des $h_{j}$ et $h_{\mathrm{j}}$ dans $C$. Identifiant les termes dominants de (P) il vient que $Q\left(X_{0}, X_{1}, \ldots, X_{i}\right) / c_{j 0}\left(X_{0}\right)$ vérifie aussi $(\mathbf{P})$ avec $R\left(X_{0}\right)=1$. Les termes de degrés 
inférieurs à 1 de chaque côté de $(\mathbf{P})$ devant être identiques, il suit:

$$
\sum_{j=1}^{i} h_{j} X_{j}=\sum_{j=1}^{i} h_{j}\left(X_{j}-\frac{X_{0}^{j}}{\left(1-T X_{0}\right)^{j}}\right)+H\left(X_{0}\right),
$$

où $H\left(X_{0}\right)$ a un pôle d'ordre multiple de $p$ en $X_{0}=1 / T$ ce qui contredit le fait que les $h_{j}$ sont non tous nuls et que $i \leq p-1$.

Nous utiliserons également la fonction $g(z)$, analytique dans le disque non circonférencié de centre 0 et de rayon 1 , définie par

$$
g(z)=\prod_{h=1}^{\infty}\left(1-T z^{q^{h}}\right) .
$$

Cette fonction a une infinité de zéros et est donc transcendante sur $C(z)$ ([D3]). Prouvons comme corollaire au Théorème 3 , l'énoncé suivant.

COROLlAIRE. Les p fonctions: $g(z), f_{i}(z)(1 \leq i \leq p-1)$ sont algébriquement indépendantes sur $C(z)$.

PREUVE. Si le résultat du corollaire n'est pas çorrect, il existe un polynôme $P \in$ $C\left[Y, X_{0}, X_{1}, \ldots, X_{p-1}\right]$ irréductible et de degré minimal $D \geq 1$, en $Y$, tel que pour tout $z$ dans le disque de convergence:

$$
P\left(g(z), z, f_{1}(z), \ldots, f_{p-1}(z)\right)=0
$$

et donc aussi:

$$
P\left(\left(1-T z^{q}\right) g(z), z^{q}, f_{1}(z)-\frac{z}{(1-T z)}, \ldots, f_{p-1}(z)-\frac{z^{p-1}}{(1-T z)^{p-1}}\right)=0 .
$$

Il s'ensuit comme précédemment, l'existence d'un polynôme $R \in C\left[Y, X_{0}, X_{1}, \ldots\right.$, $\left.X_{p-1}\right]$ et d'un entier $N$ tels que:

$$
\begin{aligned}
R\left(Y, X_{0}, X_{1}, \ldots, X_{p-1}\right) P\left(Y, X_{0}, X_{1}, \ldots, X_{p-1}\right) & \\
= & \left(1-T X_{0}\right)^{N} P\left(\left(1-T X_{0}^{q}\right) Y, X_{0}^{q}, X_{1}-\frac{X_{0}}{\left(1-T X_{0}\right)}, \ldots,\right. \\
& \left.X_{p-1}-\frac{X_{0}^{p-1}}{\left(1-T X_{0}\right)^{p-1}}\right),
\end{aligned}
$$

puis on voit, pour des raisons de degré, que $R \in C\left[X_{0}\right]$. Ecrivons $P$ comme un polynôme en $Y: P(Y)=\sum_{i=0}^{D} a_{i} Y^{i}$. L'identification des termes en $Y^{i}$ dans la relation précédente, prouve que $a_{i}$ satisfait la relation $(\mathbf{P})$ de la preuve du Théorème 3 , donc que $a_{i} \in C\left[X_{0}\right]$. Ceci contredit le fait que $g$ est une fonction transcendante. 
Passons enfin à la preuve du Théorème 1 .

PREUVE DU ThÉORÈME 1. Comme déjà vu dans ([D3]), la période de l'exponentielle $\left(T-T^{q}\right)^{1 /(q-1)} \pi$ est eǵale à $(-T)^{1 /(q-1)} \prod_{i=1}^{\infty}\left(1-T^{1-q^{i}}\right)^{-1}$, par conséquent $\pi$ est un multiple algébrique de $[g(1 / T)]^{-1}$. On voit également que la dérivée logarithmique:

$$
\frac{\pi^{\prime}}{\pi}=\sum_{h=1}^{\infty} \frac{1}{T^{q^{h}}-T}
$$

De là, il découle par dérivation que pour tout entier $i, 1 \leq i \leq p-1$, il existe une fraction rationnelle $S_{i} \in F_{q}\left(X_{0}, X_{1}, \ldots, X_{i-1}\right)$ telle que

$$
\frac{\pi^{(i)}}{\pi}+S_{i}\left(\pi, \pi^{\prime}, \ldots, \pi^{(i-1)}\right)=\sum_{h=1}^{\infty} \frac{(i-1) !}{\left(T^{q^{h}}-T\right)^{i}},
$$

avec la convention usuelle $0 !=1$.

Il s'ensuit que $L=\vec{k}\left(g(1 / T), \sum_{h=1}^{\infty} 1 /\left(T^{q^{h}}-T\right), \ldots, \sum_{h=1}^{\infty} 1 /\left(T^{q^{h}}-T\right)^{p-1}\right)$ est un sous-corps de $\bar{k}\left(\pi, \ldots, \pi^{(p-1)}\right)$, il suffit donc de prouver que $L$ est de degré de transcendance $p$ sur $k$. Remarquons alors que

$$
\sum_{h=1}^{\infty} \frac{1}{\left(T^{q^{h}}-T\right)^{i}}=\sum_{h=1}^{\infty} \frac{T^{-i q^{h}}}{\left(1-T^{1-q^{h}}\right)^{i}}=\left(f_{i}(z)-\frac{z^{i}}{(1-T z)^{i}}\right)_{z=1 / T},
$$

le corollaire précédent joint au Théorème 2 donne la conclusion.

\section{References}

[B1] P. G. Becker, 'Algebraic independence of the values of certain series by Mahler's method', Monatsh. Math. 4 (1992), 183-198.

[B2] —-, 'Transcendence measures for the values of generalized Mahler functions in arbitrary characteristic', Publ. Math. Debrecen 45 (1994), 269-282.

[B] D. Brownawell, 'Linear independence and divided derivatives of a Drinfeld module I', in: Proceedings of Schinzel Conference, Zakopane, Poland (Walter de Gruyter, Berlin, 1998) to appear.

[BD] D. Brownawell and L. Denis, 'Linear independence and hyperderivatives on Drinfeld modules II', Proc. Amer. Math. Soc., to appear.

[C] L. Carlitz, 'On certain functions connected with polynomials in a Galois field', Duke Math. J. 1 (1935), 137-168.

[D1] L. Denis, 'Dérivées d'un module de Drinfeld et transcendance', Duke Math. J. 80 (1995), 1-13.

[D2] — - 'Indépendance algébrique en caractéristique deux', J. Number Theory 66 (1997), 183-200.

[D3] —- 'Indépendance algébrique de différents $\pi$ ', C. R. Acad. Sci. Paris 327 (1998), 711-714.

[N] K. Nishioka, Mahler functions and transcendence, Lecture Notes in Math. 1631 (Springer, Berlin, 1987). 
[P] P. Philippon, 'Critères pour l'indépendance algébrique dans les anneaux Diophantiens', C. R. Acad. Sci. Paris 315 (1992), 511-515.

Université des Sciences et Technologies de Lille

UFR de Mathématiques

59655 Villeneuve d'Asq

France

e-mail: denis@univ-Lille1.fr 